Bull. Austral. Math. Soc.

VOL. 58.(1998) [137-145]

\title{
ON ISOMORPHISMS OF CONNECTED CAYLEY GRAPHS, III
}

\author{
Cai Heng Li
}

For a finite group $G$ and a subset $S$ of $G$ which does not contain the identity of $G$, we use $\operatorname{Cay}(G, S)$ to denote the Cayley graph of $G$ with respect to $S$. For a positive integer $m$, the group $G$ is called a (connected) $m$-DCI-group if for any (connected) Cayley graphs $\operatorname{Cay}(G, S)$ and $\operatorname{Cay}(G, T)$ of out-valency at most $m, S^{\sigma}=T$ for some $\sigma \in \operatorname{Aut}(G)$ whenever $\operatorname{Cay}(G, S) \cong \operatorname{Cay}(G, T)$. Let $p(G)$ be the smallest prime divisor of $|G|$. It was previously shown that each finite group $G$ is a connected $m$-DCI-group for $m \leqslant p(G)-1$ but this is not necessarily true for $m=p(G)$. This leads to a natural question: which groups $G$ are connected $p(G)$-DCI-groups? Here we conjecture that the answer of this question is positive for finite simple groups, that is, finite simple groups are all connected 2-DCI-groups. We verify this conjecture for the linear groups $\operatorname{PSL}(2, q)$. Then we prove that a nonabelian simple group $G$ is a 2 -DCI-group if and only if $G=\mathrm{A}_{5}$.

\section{INTRODUCTION}

For a finite group $G$ and a subset $S$ of $G$ which does not contain the identity of $G$, we define the Cayley graph of $G$ with respect to $S$ to be the directed graph $\operatorname{Cay}(G, S)$ with vertex set $G$ and edge set $\left\{(a, b) \mid a, b \in G, b a^{-1} \in S\right\}$. A Cayley graph $\operatorname{Cay}(G, S)$ is called a $C I$-graph of $G$ if, for any $T \subseteq G, \operatorname{Cay}(G, S) \cong \operatorname{Cay}(G, T)$ implies $S^{\sigma}=T$ for some $\sigma \in \operatorname{Aut}(G)$. (CI stands for Cayley Isomorphism.) Further, for a finite group $G$ and a positive integer $m$, if every connected Cayley graph of $G$ of out-valency at most $m$ is a CI-graph, we call $G$ a connected $m$-DCI-group; while if every Cayley graph of $G$ of out-valency at most $m$ is a CI-graph, we call $G$ an $m-D C I$-group. This paper is a contribution to characterising (connected) $m$-DCI-groups.

There has been a lot of study on the problem of determining $m$-DCI-groups in the literature, see the surveys in $[1,14,18,19]$. In particular, dependent on the finite simple group classification, a good description of 1-DCI-groups was obtained by Zhang [24], and further, a classification of $m$-DCl-groups for $m \geqslant 2$ was obtained by Praeger, Xu and the author [16]. One of reasons for investigating $m$-DCI-groups is to decide isomorphic

Received 12th January, 1998

This work forms a part of an ARC small grant project. The author thanks Professor Cheryl Praeger for her helpful suggestions on the work of the paper.

Copyright Clearance Centre, Inc. Serial-fee code: 0004-9729/98 \$A2.00+0.00. 
classes of Cayley graphs. It is in general very difficult to determine whether or not two given Cayley graphs are isomorphic. However, if $G$ is an $m$-DCI-group then two Cayley graphs $\operatorname{Cay}(G, S)$ and $\operatorname{Cay}(G, T)$ of valency at most $m$ are isomorphic if and only if $S$ and $T$ are conjugate under $\operatorname{Aut}(G)$. The latter is often much easier to determine than the former. By the definition, a Cayley graph $\operatorname{Cay}(G, S)$ is connected if and only if $S$ is a generating subset of $G$, that is, $\langle S\rangle=G$. Since a disconnected Cayley graph is a vertex disjoint union of isomorphic connected Cayley graphs of smaller order, in some sense only the isomorphism problem for connected Cayley graphs needs to be considered (see for example [22]). There have been some results on the problem of determining connected $m$-DCI-groups. Delorme, Favaron and Maheo [6] proved that all Abelian groups are connected 2-DCI-groups; Xu and Meng [23] completely classified the Abelian connected 3-DCI-groups. Some more general results were obtained by the author [11, 12]. For a finite group $G$, let $p(G)$ denote the smallest prime divisor of $|G|$. It was shown in [11] that

(1) if $G$ is a finite group then $G$ is a connected $m$-DCI-group for $m \leqslant p(G)-1$.

Further, it was also proved that the conclusion (1) can be extended to $m=p(G)$ if $G$ is Abelian. But the author [13] constructed an infinite family of groups which are not connected 2-DCI-groups, so (1) cannot be extended to $m=p(G)$ for arbitrary groups. A natural question arises here:

QUESTION 1.1. Which groups $G$ are connected $p(G)$-DCI-groups?

We are inclined to believe that the answer of this question is positive for most groups, and in particular for simple groups, namely,

ConjeCture 1.2. Finite simple groups are all connected 2-DCI-groups.

We show that this conjecture is true for the simple groups $\operatorname{PSL}(2, q)$.

Theorem 1.3. Let $G=\operatorname{PSL}(2, q)$, and let $\Gamma$ be a connected Cayley graph of $G$ of valency 2. Then

(i) $G \lessgtr$ Aut $\Gamma$;

(ii) $G$ is a connected 2-DCI-group.

The argument in the proof of this theorem given in Section 3 might be modified to prove that Conjecture 1.2 holds for some other families of nonabelian simple groups, for example, Suzuki groups $\mathrm{Sz}(q)$. But we do not think it can be used to prove Conjecture 1.2 completely. The next result gives a complete classification of simple 2-DCI-groups.

THEOREM 1.4. A finite nonabelian simple group $G$ is a 2-DCI-group if and only if $G=\mathrm{A}_{5}$.

It is proved in [16, Theorem 1.2] that if $G$ is an insoluble 2-DCI-group then $G=U \times V$ where $V=A_{5}$ or $\operatorname{PSL}(2,8)$ such that $(|U|,|V|)=1$ and all Sylow subgroups of $U$ are 
homocyclic. Further, it is easy to see that any characteristic subgroup of $G$ is also a 2-DCI-group. Thus we have an improvement on a result of [16]:

Corollary 1.5. Suppose that $G$ is an insoluble 2-DCI-group. Then $G=$ $U \times A_{5}$ for some Abelian group $U$ such that $(|U|, 2.3 .5)=1$ and all Sylow subgroups of $U$ are homocyclic.

After we draw together some preliminary results in Section 2, we prove Theorem 1.3 in Section 3. Then in Section 4, we prove Theorem 1.4.

\section{Preliminaries}

This section collects several preliminary results which will be used. The notation and terminology used in the paper are standard (see, for example, [4]). For convenience, we list some of them here. Let $\mathbb{Z}_{n}$ denote the cyclic group of order $n$. For a group $G$ and its a subgroup $H,|G: H|(=|G| /|H|)$ denotes the index of $H$ in $G$. For two groups $G$ and $H, G \rtimes H$ denotes a semidirect product of $G$ by $H$. Let $\Gamma$ be a graph and let Aut $\Gamma$ be the full automorphism group of $\Gamma$. Let $\Gamma(\alpha)$ be the neighbourhood of the vertex $\alpha$ of $\Gamma$, namely the set of all vertices of $\Gamma$ which are joined to $\alpha$. For a subgroup $G$ of Aut $\Gamma$, the graph $\Gamma$ is said to be $G$-vertex-transitive or $G$-arc-transitive if $G$ acts transitively on the set of vertices or on the set of arcs of $\Gamma$, respectively. For $\alpha \in V \Gamma$, let $G_{\alpha}$ denote the stabiliser of $\alpha$ in $G$, and let $G_{\alpha}^{\Gamma(\alpha)}$ denote the permutation group on $\Gamma(\alpha)$ induced by $G_{a}$. For a finite group $G$ and its a subgroup $H$, we can construct arc-transitive graphs as follows (see [20] and [17]):

DEFINITION 2.1. Let $G$ be a finite group. Suppose that there are a subgroup $H<G$ and an element $g \in G$. A graph $\Gamma=\Gamma(G, H, g)$ is defined as $V \Gamma=\{x H \mid x \in G\}$ and $(x H, y H)$ is an arc of $\Gamma$ if and only if $y x^{-1} \in H g H$.

Such a graph has the following properties.

LEMmA 2.2. ([20] and [17, Theorems 1 and 2].) Let $\Gamma=\Gamma(G, H, g)$ be a graph defined as in Definition 2.1. Then

(1) $G \leqslant$ Aut $\Gamma$ and $G_{\alpha}=H$, where $\alpha$ is a vertex of $\Gamma$ corresponding $H$;

(2) $\Gamma$ is a $G$-arc-transitive graph of valency $\left|H: H \cap H^{s}\right|$;

(3) $\Gamma$ is connected if and only if $\langle H, g\rangle=G$.

For a Cayley graph $\Gamma$ of $G$, the normaliser of $G$ in Aut $\Gamma$ is often useful for characterising the structure of $\Gamma$.

Lemma 2.3. (See [7, Lemma 2.1]) Let $G$ be a finite group and $S$ a subset of $G$, let $A:=\operatorname{AutCay}(G, S)$ and $\operatorname{Aut}(G, S):=\left\{\sigma \in \operatorname{Aut}(G) \mid S^{\sigma}=S\right\}$. Then $\mathrm{N}_{A}(G)=$ $G \rtimes \operatorname{Aut}(G, S)$.

Next we have a criterion for a Cayley graph to be a CI-graph, which was obtained by Babai [3], and also by Alspach and Parsons [2]. 
ThEOREM 2.4. Let $\Gamma$ be a Cayley graph of a finite group $G$, and let $\operatorname{Sym}(G)$ denote the symmetric group on $G$. Then $\Gamma$ is a CI-graph if and only if, for any $\tau \in \operatorname{Sym}(G)$ with $G^{\tau} \leqslant$ Aut $\Gamma$, there is $\sigma \in$ Aut $\Gamma$ such that $G^{\sigma}=G^{\tau}$.

A graph $\Gamma$ is called a cover of a graph $\Sigma$ if there is a surjection $\phi$ from the vertex-set of $\Gamma$ to the vertex-set of $\Sigma$ which preserves adjacency and is such that, for each vertex $\alpha$ of $\Gamma$, the set $\Gamma(\alpha)$ and $\Sigma\left(\alpha^{\phi}\right)$ of neighbours of $\alpha$ and $\alpha^{\phi}$ in $\Gamma$ and $\Sigma$ respectively have the same size. In this case, $\Sigma$ is called a quotient graph of $\Gamma$. If $\Gamma$ is a $G$-vertex-transitive graph and $N$ is a normal subgroup of $G$ which acts regularly on $V \Gamma$, then $\Gamma$ has a quotient graph $\Gamma_{N}$, for which $V \Gamma_{N}$ is the set of all $N$-orbits on $V \Gamma$, and two vertices $U, V \in V \Gamma_{N}$ are adjacent in $\Gamma_{N}$ if and only if there exist $\beta \in U$ and $\alpha \in V$ which are adjacent in $\Gamma$. The graph $\Gamma_{N}$ is said to be induced by $N$.

LEMma 2.5. Let $\Gamma$ be a connected $G$-vertex-transitive di-graph where $G \leqslant$ Aut $\Gamma$. Let $N$ be a normal subgroup of $G$ and $\alpha \in V \Gamma$. Assume that $G_{\alpha}^{\Gamma(\alpha)}$ is primitive and that $N$ is not transitive on $V \Gamma$. Then either $\Gamma$ is a cycle, or $N$ is semi-regular on $V \Gamma$ and $\Gamma_{N}$ is $G / N$-arc transitive of valency $|\Gamma(\alpha)|$.

ProOF: Suppose that $N$ is not semiregular on $V \Gamma$. Then $N_{\alpha}^{\Gamma(\alpha)} \neq 1$. Since $N_{\alpha}^{\Gamma(\alpha)} \unlhd$ $G_{\alpha}^{\Gamma(\alpha)}$, we have that $N_{\alpha}^{\Gamma(\alpha)}$ is transitive on $\Gamma(\alpha)$. Thus the quotient graph $\Gamma_{N}$ is of valency 1 , and so $\Gamma$ is a cycle. Therefore, if $\Gamma$ is not a cycle, then $N$ is semi-regular on $V \Gamma$ and it is then easy to see that $\Gamma_{N}$ is $G / N$-arc transitive of valency $|\Gamma(\alpha)|$.

\section{Proof of Theorem 1.3}

This section is devoted to proving Theorem 1.3. First we prove some simple properties of Cayley graphs of valency two.

Proposition 3.1. Let $G$ be a finite nonabelian simple group, and let $\Gamma$ be a connected $G$-vertex-transitive digraph of $G$ of valency two.

(1) Let $A \leqslant$ Aut $\Gamma$ be such that $G \leqslant A$, and let $\alpha \in V \Gamma$. Let $N$ be a normal subgroup of $A$. Then either $N$ is a 2-group such that $|N||| A_{\alpha} \mid$, or $G \leqslant N$ and if in addition $N$ is minimal normal in $A$ then $N$ is nonabelian simple and is characteristic in $A$;

(2) Suppose further that $\Gamma$ is a Cayley graph of $G$ and that $G$ is normal in Aut $\Gamma$. Then $\Gamma$ is a CI-graph.

Proof: (1) Since $G \cap N \leqslant G$ and $G$ is simple, either $G \cap N=1$, or $G \leqslant N$. Assume first that $G \cap N=1$. Then $|N|$ divides $|G N| /|G|$ which divides $|A| /|G|=\left|A_{\alpha}\right|$. Since the valency of $\Gamma$ is 2 , we have that $A_{\alpha}$ and so $N$ is a 2-group. Assume now that $G \leqslant N$. Then $N$ is insoluble and $|N: G||| A: G|=| A_{\alpha} \mid$. Further assume that $N$ is a minimal normal subgroup of $A$. Then $N=T_{1} \times \ldots \times T_{r}$ where $T_{1} \cong \ldots \cong T_{r}$ is nonabelian simple. Since $T_{i} \triangleq N, T_{i} \cap G \lessgtr G$ for all $i \in\{1, \ldots, r\}$, and hence either $T_{i} \cap G=1$ or $G \leqslant T_{i}$. If 
$T_{i} \cap G=1$ for some $i$, then $\left|\left\langle T_{i}, G\right\rangle\right|=\left|T_{i}\right||G|$ divides $|A|=\left|G A_{\alpha}\right|=|G|\left|A_{\alpha}\right| /\left|G \cap A_{\alpha}\right|$, which is a contradiction since $\left|T_{i}\right| \nmid\left|A_{\alpha}\right|$. Thus $G \leqslant T_{i}$ for each $i$. It follows that $r=1$ and so $N$ is simple. This argument also proves that $N$ is a unique insoluble minimal normal subgroup of $A$. Thus $N$ is a characteristic subgroup of $A$.

(2) Let $A=$ Aut $\Gamma$. Then $A=G \rtimes A_{\alpha}$ such that $A_{\alpha}$ is a 2-group. Let $\tau \in \operatorname{Sym}(G)$ be such that $G^{\tau} \leqslant A$. If $G^{\tau} \neq G$ then since $G^{\tau} \cap G \leqslant G^{\tau}$, we have $G^{\tau} \cap G=1$. Thus $\left\langle G, G^{\tau}\right\rangle=G \rtimes G^{\tau}$, and so $\left|G^{\tau}\right|=\left|\left\langle G, G^{\tau}\right\rangle: G\right|$ divides $|A: G|=\left|A_{\alpha}\right|$, which is a contradiction since $A_{\alpha}$ is a 2-group. Therefore, $G^{\tau}=G$, and so by Theorem 2.4, $\Gamma$ is a CI-graph.

Now we are ready to prove Theorem 1.3.

Proof of Theorem 1.3: By Proposition 3.1 (2), part (ii) follows from part (i). Thus we need only prove part (i). Suppose to the contrary that $G$ is not normal in Aut $\Gamma$. Our task in the rest is to seek a contradiction. Let $A=$ Aut $\Gamma$ and $\alpha \in V \Gamma$. Then $A=G A_{\alpha}$ such that $G \cap A_{\alpha}=1$ and $A_{\alpha}$ is a nontrivial 2-group. In particular, $G$ is a proper subgroup of $A$ of a 2-power index. If $A$ is a simple group then by a result of Guralnick $[8],(G, A)=\left(\mathrm{A}_{n-1}, \mathrm{~A}_{n}\right)$ for some $n=2^{r}$ with $r \geqslant 3$, which is not the case. Therefore, $A$ is not a simple group.

Let $M$ be the largest soluble normal subgroup of $A$. Then $M \cap G=1$, and $M$ is a 2-group (by Proposition 3.1). Let $\tilde{A}=A / M$, and let $\tilde{B}$ be a minimal normal subgroup of $\widetilde{A}$. By the maximality of $M, \widetilde{B}$ is insoluble, and therefore by Proposition 3.1(1), $\widetilde{B}$ is simple and $\tilde{G}:=M G / M \leqslant \tilde{B}$. Note that $\tilde{G}$ is a subgroup of $\tilde{B}$ of 2-power index. If $\widetilde{G} \neq \widetilde{B}$, then by $[8]$, we have that $(\widetilde{G}, \widetilde{B})=\left(\mathrm{A}_{n-1}, \mathrm{~A}_{n}\right)$ for some $n=2^{r}$ with $r \geqslant 3$, which is not the case. Thus $\tilde{G}=\tilde{B}$. Let $B$ be the full preimage of $\widetilde{B}$ under $A \rightarrow \tilde{A}$. Then $B=M \rtimes G$ and $B$ is characteristic in $A$. If $G$ centralises $M$, then $B=M \times G$ and therefore, $G$ is characteristic in $B$ and so is normal in $A$, which is a contradiction. Thus $G$ does not centralise $M$.

Take a (principal) series of subgroups of $B$ :

$$
1=M_{0}<M_{1}<\ldots<M_{t}=M<B \leqslant A,
$$

where every $M_{i} \triangleleft B$ and $M_{i} / M_{i-1}$ is a minimal normal subgroup of $B / M_{i-1}$. Since $G$ does not centralise $M$, there exists $i \in\{0,1, \ldots, t-1\}$ such that $G$ centralises $M_{i}$ but does not centralise $M_{i+1}$. We claim that $\bar{G}:=M_{i} G / M_{i}$ does not centralise $\bar{M}_{i+1}:=$ $M_{i+1} / M_{i}$. Suppose to the contrary that $\bar{G}$ centralises $\bar{M}_{i+1}$. Let $F=\left\langle M_{i+1}, G\right\rangle$. Then $\bar{F}:=F / M_{i}=\left\langle\bar{M}_{i+1}, \bar{G}\right\rangle=\bar{M}_{i+1} \times \bar{G}$. Thus $\bar{G}$ is characteristic in $\bar{F}$ and so the full preimage $M_{i} \rtimes G$ of $\bar{G}$ under $F \rightarrow \bar{F}$ is normal in $F$. Since $G$ centralises $M_{i}$, we have $M_{i} \rtimes G=M_{i} \times G$. Thus $G$ is characteristic in $M_{i} \rtimes G$ and so normal in $F$. Since $M_{i+1} \triangleleft F$ and $M_{i+1} \cap G=1$, we have that $F=M_{i+1} \times G$ so $G$ centralises $M_{i+1}$, which is a contradiction. Therefore, $\bar{G}$ does not centralise $\bar{M}_{i+1}$. 
For convenience, write $\bar{M}_{i+1}$ as $X, \bar{G}$ as $Y$ and the quotient graph $\Gamma_{M_{i}}$ (induced by $M_{i}$ ) as $\Sigma$, respectively, and set $Z:=X \rtimes Y$. It follows from Proposition 3.1 that $X=\mathbb{Z}_{2}^{n}$ for some $n \geqslant 1$. Further, $X$ is a minimal normal subgroup of $Z, Y(\cong G)$ does not centralise $X$ and acts transitively on $V \Sigma$, and by Lemma 2.5, $\Sigma$ is a $Z$-arc-transitive digraph of valency 2. Therefore, $Y$ is irreducible on $X$. If $X$ is not semiregular on $V \Sigma$ then the quotient graph $\Sigma_{X}$ induced by $X$ is a cycle and $\bar{Z}:=Z / X \cong \operatorname{PSL}(2, q)$ is transitive on $V \Sigma$, which is not possible. Hence $X$ is semiregular on $V \Sigma$. Since $Y$ is transitive on $V \Sigma,|X||| Y \mid$.

Suppose first that $q=2^{r}$ for some $r \geqslant 1$. Then a Sylow 2-subgroup of $Y(\cong$ $\operatorname{PSL}(2, q))$ is isomorphic to $\mathbb{Z}_{2}^{r}$ and any two Sylow 2-subgroups have trivial intersection (see for example $\left[\mathbf{2 1}\right.$, p. 417] and $\left[\mathbf{9}\right.$, p. 295]). Let $\bar{Z}=Z / X$ and $\bar{\Sigma}=\Sigma_{X}$. Then $\bar{Z} \cong$ $\operatorname{PSL}(2, q)$ and $\bar{\Sigma}$ is a $\bar{Z}$-arc-transitive digraph of valency 2 (by Lemma 2.5 ). Thus $H:=\bar{Z}_{\beta}$ is a 2-subgroup of $\bar{Z}$ where $\beta \in V \bar{\Sigma}$ and $\bar{Z}_{\beta}$ is the stabiliser of $\beta$ in $\bar{Z}$. Now $\bar{Z} \cong Y$ and $Y$ is transitive on $V \Sigma$. Since $\left|\bar{Z}_{\beta}\right|=|\bar{Z}| /\left|V \Sigma_{X}\right|=|\bar{Z}|(|V \Sigma| /|X|)=|X| \cdot|Y| /|V \Sigma|$, we have that $|X||| \bar{Z}_{\beta}|=| H \mid$. By Lemma 2.2, there exists $g \in \bar{Z}$ such that $\left|H: H^{g} \cap H\right|=2$. Since any two Sylow 2-subgroups of $\bar{Z}$ have trivial intersection, $\bar{Z}$ has a unique Sylow 2-subgroup $P$ containing $H$ as a subgroup and either $g$ normalises $P$, or $P^{g} \cap P=1$. If $P^{g} \cap P=1$ then $H^{g} \cap H=1$ and so $|H|=2$. Thus $X \cong \mathbb{Z}_{2}$ and so $Y$ centralises $X$, which is a contradiction. If $g$ normalises $P$, then $\bar{Z}=\langle H, g\rangle \leqslant\langle P, g\rangle<\bar{Z}$, which is also a contradiction.

Now suppose that $q$ is odd, and let $s$ be the order of a Sylow 2-subgroup of $Y$. It is easy to see that if $q \equiv 1(\bmod 4)$ then $s$ divides $q-1$, and if $q \equiv-1(\bmod 4)$ then $s$ divides $q+1$. Thus in either case $s \leqslant q+1$. Since $|X|$ divides $|Y|, 2^{n}=|X| \leqslant s \leqslant q+1$. Since $Y$ is irreducible on $X$, by [10, Theorem 5.3.9], we have $n \geqslant(q-1) / 2$. Therefore, $2^{(q-1) / 2} \leqslant 2^{n} \leqslant q+1$. Solving this inequality, we find $q \leqslant 7$, that is, $q=5$ or 7 . If $q=5$ then $s=4$ and so $|X| \mid 4$ but $Y \notin \mathrm{GL}(2,2)$, which is a contradiction. Therefore, we have $q=7$ and $Z \cong \mathbb{Z}_{2}^{3} \rtimes \operatorname{PSL}(2,7)$. Let $\bar{Z}=Z / X$ and $\bar{\Sigma}=\Sigma_{X}$. Then $\left|\bar{Z}_{\beta}\right|=8$ where $\beta \in V \Sigma$. Thus $\bar{\Sigma}$ is of order 21 and 3-arc-transitive. By [4, Lemma 16.3], $\bar{\Sigma}$ is a Cayley graph, that is, $\bar{\Sigma}=\operatorname{Cay}\left(\mathbb{Z}_{7} \rtimes \mathbb{Z}_{3}, S\right)$ for some subset $S$ of $\mathbb{Z}_{7} \rtimes \mathbb{Z}_{3}$. Since $\bar{\Sigma}$ is 3-arc-transitive, it follows that $S$ consists of elements of order 7 . But such an $S$ is such that $\langle S\rangle \cong \mathbb{Z}_{7}$, which is a contradiction to the connectivity of $\bar{\Sigma}$. This completes the proof of the theorem.

\section{Proof of Theorem 1.4}

Here we shall prove that the only nonabelian simple 2-DCI-group is $A_{5}$. First we consider the alternating group $\mathbf{A}_{4}$.

LEMma 4.1. Let $G=\mathrm{A}_{4}$. Then

(i) any connected Cayley graph of $G$ of valency 2 is not 2-arc transitive; 


\section{(ii) $G$ is a $2-D C I$-group.}

Proof: It is known that $\operatorname{Aut}(G)=\mathrm{S}_{4}$, and so it follows that $G$ is a 1-DCI-group and that all undirected Cayley graphs of $G$ of valency 2 are CI-graphs. Thus we only need to consider subsets which have the form $S=\{a, b\}$ such that $|S|=2$ and $\langle S\rangle=G$. Let $\Gamma=\operatorname{Cay}(G, S)$, and let $A=\operatorname{Aut} \Gamma$ and $\alpha \in V \Gamma$. If $\Gamma$ is not arc-transitive, then $A_{\alpha}=1$ and so $\Gamma$ is a CI-graph by Theorem 2.4. If $\Gamma$ is arc-transitive, then it follows that $o(a)=o(b)=3$. Now it is easy to see that $\Gamma$ is not 2-arc-transitive. Thus $\left|A_{\alpha}\right|=2$ and so $|A|=\left|G A_{\alpha}\right|=2|G|$. For any $\tau \in \operatorname{Sym}(G)$ such that $G^{\tau} \leqslant A$, if $G^{\tau} \neq G$ then $\left|G^{\tau} \cap G\right|=\left(|G|\left|G^{\tau}\right|\right) /\left|G G^{\tau}\right|=|G| / 2$, which is a contradiction since $G$ does not have a subgroup of index 2. Thus $G^{\tau}=G$ and $\Gamma$ is a CI-graph by Theorem 2.4, so $G$ is a 2-DCI-group.

Proof of Theorem 1.4: Assume that $G$ is a nonabelian simple 2-DCI-group. By [16] (or [15]), $G=\mathrm{A}_{5}$ or PSL(2,8). Suppose that $G=\operatorname{PSL}(2,8)$. Then $G$ has a subgroup $H \cong \operatorname{AGL}\left(1,2^{3}\right)=\mathbb{Z}_{2}^{3} \rtimes \mathbb{Z}_{7}$. By [13, Corollary 2.7], $\operatorname{AGL}\left(1,2^{3}\right)$ is not a connected 2DCI-group. Hence there exist two Cayley graphs $\operatorname{Cay}(G, S)$ and $\operatorname{Cay}(G, T)$ of valency 2 such that $\langle S\rangle=\langle T\rangle=H, \operatorname{Cay}(H, S) \cong \operatorname{Cay}(H, T)$ and $S$ is not conjugate in $\operatorname{Aut}(H)$ to $T$. It follows that $\operatorname{Cay}(G, S) \cong \operatorname{Cay}(G, T)$. If there exists $\sigma \in \operatorname{Aut}(G)$ such that $S^{\sigma}=T$, then $H^{\sigma}=H$ and so $\sigma$ induces an automorphism $\tau$ of $H$ which sends $S$ to $T$, which is a contradiction. So PSL $(2,8)$ is not a 2-DCI-group, and therefore, $G=\mathrm{A}_{5}$.

Conversely, we need to verify that $\mathrm{A}_{5}$ is really a 2-DCI-group. Set $G:=\mathrm{A}_{5}$, and let $\Gamma:=\operatorname{Cay}(G, S)$ be of valency at most 2. By [15, Theorem 3], if $\Gamma$ is undirected then $\Gamma$ is a CI-graph, and on the other hand, by Theorem 1.3, if $\Gamma$ is connected then $\Gamma$ is a CI-graph. Further, since $\operatorname{Aut}(G)=\mathrm{S}_{5}$, any two elements of $G$ of the same order are conjugate in $\operatorname{Aut}(G)$, so if $|S|=1$ then $\Gamma$ is a CI-graph. Thus we may assume that $S \neq S^{-1},|S|=2$ and $\langle S\rangle<G$. Let $H$ be a maximal subgroup of $G$ which contains $S$. By the Atlas [5], $H \cong \mathrm{A}_{4}, \mathrm{D}_{10}$ or $\mathrm{S}_{3}$, and any maximal subgroup of $G$ of order $|H|$ is conjugate to $H$. If $\langle S\rangle\left\langle H\right.$ then $\langle S\rangle \cong \mathbb{Z}_{5}$ (since at this moment $S \neq S^{-1}$ and $|S|=2$ ). Since all elements of order 5 are conjugate in $\operatorname{Aut}(G)$, it follows that $\Gamma$ is a CI-graph of $G$. Thus we may further assume that $\langle S\rangle=H$. Then by [3] and Lemma $4.1, \Gamma$ is a CI-graph of $H$. Let $T \subset G$ be such that $\operatorname{Cay}(G, S) \cong \operatorname{Cay}(G, T)$. Then $\langle T\rangle \cong H$. Since all subgroups of $G$ of order $|H|$ are conjugate to $H$, there exists $g \in G$ such that $T^{g} \subset H$. Thus $\operatorname{Cay}(H, S) \cong \operatorname{Cay}\left(H, T^{g}\right)$. Since $\Gamma$ is a CI-graph of $H$, there exists $\alpha \in \operatorname{Aut}(H)$ such that $S^{\alpha}=T^{g}$. By the Atlas [5], Aut $(H) \cong \mathbf{N}_{\mathrm{Aut}(G)}(H) / \mathbf{C}_{\mathrm{Aut}(G)}(H)$, and hence $S^{\sigma}=T^{g}$ for some $\sigma \in \mathbf{N}_{\text {Aut }(G)}(H)$. Therefore, $S$ is conjugate in $\operatorname{Aut}(G)$ to $T$, and so Cay $(G, S)$ is a CI-graph of $G$. Hence $\mathrm{A}_{5}$ is a 2-DCI-group, and this completes the proof of Theorem 1.4. 


\section{REFERENCES}

[1] B. Alspach, 'Isomorphisms of Cayley graphs on Abelian groups', in Graph Symmetry: Algebraic Methods and Applications 497, NATO Adv. Sc. Inst. Ser. C (Kluwer Acad. Publ., Dordrecht, 1997), pp. 1-23.

[2] B. Alspach and T.D. Parsons, 'Isomorphisms of circulant graphs and digraphs', Discrete Math. 25 (1979), 97-108.

[3] L. Babai, Isomorphism problem for a class of point-symmetric structures, Acta Math. Acad. Sci. Hungar. 29 (1977), 329-336.

[4] N. Biggs, Algebraic graph theory (Cambridge University Press, New York, 1992).

[5] J.H. Conway, R.T. Curtis, S.P. Norton, R.A. Parker and R.A. Wilson, Atlas of finite groups (Clarendon Press, Oxford, 1985).

[6] C. Delorme, O. Favaron and M. Maheo, 'Isomorphisms of Cayley multigraphs of degree 4 on finite Abelian groups', European J. Combin. 13 (1992), 59-61.

[7] C.D. Godsil, 'On the full automorphism group of a graph', Combinatorica 1 (1981), 243-256.

[8] R. Guralnick, 'Subgroups of prime power index in a simple group', J. Algebra 81 (1983), 304-311.

[9] B. Huppert and N. Blackburn, Finite Groups III (Springer-Verlag, New York, 1982).

[10] P.B. Kleidman and M.W. Liebeck, The subgroup structure of the finite classical groups, London Math. Soc. Lecture Notes (Cambridge University Press, New York, Sydney, 1990).

[11] C.H. Li, 'On isomorphisms of connected Cayley graphs', Disc. Math. 78 (1998), 109-122.

[12] C.H. Li, 'Isomorphisms of Cayley digraphs of Abelian groups', Bull. Austral. Math. Soc. 57 (1998), 181-188.

[13] C.H. Li, 'On isomorphisms of connectd Cayley graphs, II', J. Combin. Theory (B) (to appear).

[14] C.H. Li, On isomorphisms of finite Cayley graphs - a survey, (UWA Research Report, 1998/8) (University of Western Australia, Australia).

[15] C.H. Li and C.E. Praeger, 'The finite simple groups with at most two fusion classes of every order', Comm. Algebra 24 (1996), 3681-3704.

[16] C.H. Li, C.E. Praeger and M.Y. Xu, 'Isomorphisms of finite Cayley digraphs of bounded valency', J. Combin. Theory (B) (to appear).

[17] P. Lorimer, 'Vertex-transitive graphs: symmetric graphs of prime valency', J. Graph Theory 8 (1984), 55-68.

[18] P.P. Pàlfy, 'Isomorphism problem for relational structures with a cyclic automorphism', European J. Combin. 8 (1987), 35-43.

[19] C.E. Praeger, 'Finite transitive permutation groups and finite vertex-transitive graphs', in Graph symmetry: algebraic methods and applications 497, NATO Adv. Sc. Inst. Ser.C (Kluwer Acad., Dordrecht, 1997), pp. 277-318.

[20] G.O. Sabidussi, 'Vertex-transitive graphs', Monatsh. Math. 68 (1964), 426-438.

[21] M. Suzuki, Group theory I (Spring-Verlag, Berlin, Heidelberg, New York, 1982).

[22] M.Y. Xu, 'Half-transitive graphs of prime-cube order', J. Algebric Combin. 1 (1992), 275-282. 
[23] M.Y. Xu and J.X. Meng, 'Weakly 3-DCI Abelian groups', Australas, J. Combin. 13 (1996), 49-60.

[24] J.P. Zhang, 'On finite groups all of whose elements of the same order are conjugate in their automorphism groups', J. Algebra 153 (1992), 22-36.

Department of Mathematics

University of Western Australia

Nedlands WA 6907

Australia 